\title{
«Off label use»: nouvelles recommandations
}

Swissmedic

Le groupe de travail mis en place par Swissmedic et par le Comité directeur de l'Association des pharmaciens cantonaux a présenté son rapport final sur l'utilisation des médicaments au sens de l'«off label use». La tâche qui incombait à ce groupe de travail consistait à remédier aux incertitudes juridiques qui planent sur l'utilisation des médicaments dans des indications thérapeutiques qui ne figurent pas dans l'information professionnelle sur le médicament approuvée par Swissmedic. En effet, les dispositions légales actuelles suscitent de nombreuses questions de la part des médecins qui prescrivent des médicaments, des pharmaciens qui les remettent, et des patients.

Plutôt que d'envisager la problématique de l'off label use isolément, le groupe de travail, qui regroupait des représentants des cantons, de Swissmedic et des pharmacies d'hôpitaux, s'est attaché à définir les critères qui différencient cette approche de l'utilisation de médicaments qui ne sont pas autorisés en Suisse («unlicensed use»), ou qui sont dispensés de l'autorisation par Swissmedic au sens de l'article 9, alinéa 2 de la loi sur les produits thérapeutiques. Le groupe de travail s'est rendu compte qu'il y avait un grand besoin d'éclaircissement dans les milieux intéressés (autorités, professionnels de la santé et patients). Par conséquent, ces recommandations ont été rédigées en mettant particulièrement l'accent sur les responsabilités des médecins et pharmaciens concernés ainsi que sur celles des autorités cantonales et fédérales responsables de la mise en œuvre de la loi sur les produits thérapeutiques.

La version complète du rapport établi par le groupe de travail off label use sous la présidence de Viviane Fahr, pharmacienne cantonale de Bâle-Ville, est disponible sur le site web de Swissmedic (www.swissmedic.ch/publications.asp, rubrique «Autres publications», sous le titre suivant: «Recommandations de l'Association des pharmaciens cantonaux et de Swissmedic au sujet de l'utilisation des médicaments au sens de $l^{\prime}$ ‘off-label use»»). Vous y trouverez les définitions des concepts d' $\mathrm{d}^{\prime}$ off label use» et $\mathrm{d}^{\prime}$ '«unlicensed use», des précisions quant au devoir de diligence qui incombe aux médecins qui prescrivent des médicaments et aux pharmaciens qui les remettent, et la description des sanctions infligées par les autorités en cas de violation de ce devoir. 